\title{
5-DOF Manipulation of an Untethered Magnetic Device in Fluid using a Single Permanent Magnet
}

\author{
Arthur W. Mahoney and Jake J. Abbott
}

\begin{abstract}
This paper presents a three degree-of-freedom (3DOF) closed-loop position and 2-DOF open-loop orientation control method for an untethered mockup magnetic capsule endoscope in fluid with a single permanent magnet positioned by a commercial robotic manipulator and a 3-DOF capsuleposition localization system. Using traditional methods known to roboticists, we study the kinematics of untethered magnetic manipulation using a single permanent magnet as the endeffector of a robot manipulator. We present a control method that maintains 5-DOF control of a magnetic capsule when the robot manipulator is not near a kinematic singularity, and seamlessly enables a capsule's position to be controlled when the manipulator nears a kinematic singularity by sacrificing control over the capsule's orientation. We demonstrate the method's robustness to a control rate of $25 \mathrm{~Hz}$, reduced localization rates down to $30 \mathrm{~Hz}$, and the presence of manipulator singularities. 5 DOF manipulation of an untethered device has been previously demonstrated by electromagnetic systems only. This work has applications for robotic capsule endoscopy of a fluid-distended stomach.
\end{abstract}

\section{INTRODUCTION}

Untethered devices that derive their power from externally applied magnetic fields have been an active area of research due to their potential for accessing hard-to-reach areas of the human body [5, 27]. These devices, which consist of a magnetic body rigidly attached to a functional structure, are particularly promising for use in the gastrointestinal (GI) system, where an untethered magnetic capsule endoscope could be used to actively image the GI system, making current gastrointestinal screening procedures faster, safer, and less invasive [27]. Untethered magnetic devices range in size from the microscale to the mesoscale and are being applied to surgical tasks in the eye [22], in vasculature [6], and other hardto-reach areas of the human body [5]. Untethered magnetic devices are often viewed as end-effectors to a larger robotic system that could consist of an arrangement of electromagnets or a permanent magnet positioned in space.

There exist a variety of methods for employing magnetic force and/or torque to propel and control untethered magnetic devices. Irrespective to the form of propulsion, an untethered device containing a single permanent magnet can be controlled with at most five degrees-of-freedom (5-DOF), without additional externally applied nonmagnetic forces or torques. To date, three electromagnetic systems have been developed with the ability to perform 5-DOF magnetic manipulation: the OctoMag [22] and Magnetecs [6] systems consist of eight ferromagnetic-core electromagnets arranged around a hemisphere and sphere, respectively, directed toward the manipulation workspace, and a system developed by Olym-

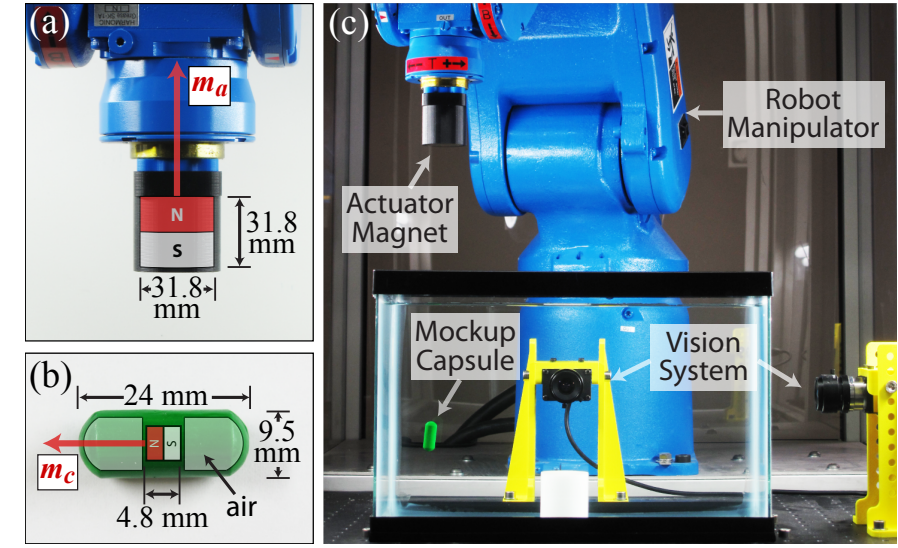

Fig. 1. The axially magnetized actuator magnet (a) is used to wirelessly control a mockup capsule endoscope (b), which is shown levitating in a waterfilled tank (c) using a 6-DOF robotic manipulator and vision localization system. Note the large distance between the actuator magnet and the capsule.

pus and Siemens, consisting of 12 electromagnets through which a patient is positioned, for the control of a magnetic capsule endoscope in a fluid-distended stomach [16]. 6-DOF electromagnetic control of an untethered device containing two permanent magnets has been demonstrated in [25]. The work presented in this paper focuses on the control of devices that contain one permanent magnet.

Compared to electromagnetic actuation systems, permanentmagnet systems are gaining attention for their ability to generate magnetic fields that can apply clinically relevant forces and torques to a magnetic device in a compact form-factor. Whereas electromagnetic systems can be designed to generate fields that are easy to control, permanent-magnet systems (particularly those that employ a single permanent magnet) have been constrained by the complex geometry of the dipole field that they produce. In similar work, a permanent magnet has been positioned both by hand and with a robot manipulator for the control of an untethered capsule endoscope, but with 4-DOF control (i.e., 2-DOF orientation control and 2-DOF position control since the endoscope always contacts a tissue surface) [10, 12, 13, 15, 26]. Permanent-magnet systems have also been used to actuate untethered devices by rolling [2, 4, 14, 24, 28] and helical propulsion [4, 17].

In this paper, we present a method for 3-DOF position and 2-DOF orientation control of a mockup magnetic capsule endoscope in fluid using a single actuator permanent magnet positioned above the capsule with a 6-DOF commercial robotic manipulator, with a target application of stomach capsule 
endoscopy (the same as the Olympus-Siemens system). The magnetic force controls the total force (the sum of forces due to gravity, buoyancy, and the magnetics) acting on the capsule and thus the capsule's position, while the applied magnetic torque controls the capsule's heading (i.e., 2-DOF pointing orientation). The magnetic force and torque are controlled by adjusting the position and orientation of the actuator magnet, using the 3-DOF capsule position measured with a localization system. This paper presents a control strategy that maintains 5-DOF control of an untethered mockup capsule endoscope when the robot manipulator is not near a kinematic singularity, and sacrifices control over the capsule's heading when the robot manipulator nears a singularity in order to maintain control over the magnetic capsule's position. 5-DOF manipulation of an untethered device has been previously demonstrated by electromagnetic systems only. This work appeared in a preliminary form in [3].

In this paper, scalars are denoted by lower-case standard font (e.g., $c$ ), vectors are denoted by lower-case bold font (e.g., $\mathbf{x}$ ), and matrices are denoted by capital standard font (e.g., $M$ ). Unit vectors are denoted with the "hat" symbol (e.g., $\hat{\mathbf{x}}$ ), and time derivatives are denoted by the "dot" symbol (e.g., $\dot{\mathrm{x}}$ ).

\section{ForwARD AND DifFERENTIAL INVERSE KinEmatics}

The capsule endoscope is assumed to contain a permanent magnet, positioned at the capsule's center-of-gravity, with its dipole moment (i.e., the vector from the south to north pole) denoted by $\mathbf{m}_{\mathbf{c}} \in \mathbb{R}^{3}$ in units $\mathrm{A} \cdot \mathrm{m}^{2}$, which is assumed to be parallel to the capsule's principle axis. The actuator magnet's dipole moment is denoted by $\mathbf{m}_{\mathbf{a}} \in \mathbb{R}^{3}$ and is positioned by a robotic manipulator with at least 5-DOF; rotation of the actuator magnet about $\mathbf{m}_{\mathbf{a}}$ is not needed. The positions of the actuator and capsule endoscope magnet centers are denoted by $\mathbf{p}_{\mathbf{a}} \in \mathbb{R}^{3}$ and $\mathbf{p}_{\mathbf{c}} \in \mathbb{R}^{3}$, respectively, in units $\mathrm{m}$.

We assume that the magnetic field $\mathbf{h}\left(\mathbf{p}, \hat{\mathbf{m}}_{\mathbf{a}}\right) \in \mathbb{R}^{3}$ generated by the actuator magnet can be modeled by the pointdipole model, which is given by

$$
\mathbf{h}\left(\mathbf{p}, \hat{\mathbf{m}}_{\mathbf{a}}\right)=\frac{\left\|\mathbf{m}_{\mathbf{a}}\right\|}{4 \pi\|\mathbf{p}\|^{3}} D(\hat{\mathbf{p}}) \hat{\mathbf{m}}_{\mathbf{a}},
$$

where $\mathbf{p}=\mathbf{p}_{\mathbf{c}}-\mathbf{p}_{\mathbf{a}}$ is the vector from the center of the actuator magnet to the center of the capsule's magnet (i.e., the relative position), $D(\hat{\mathbf{p}})=3 \hat{\mathbf{p}} \hat{\mathbf{p}}^{\top}-I$, and $I \in \mathbb{R}^{3 \times 3}$ is the identity matrix. Since the magnitudes of $\mathbf{m}_{\mathbf{a}}$ and $\mathbf{m}_{\mathbf{c}}$ are constant (they are the dipole moments of permanent magnets), we express all functions of $\mathbf{m}_{\mathbf{a}}$ and $\mathbf{m}_{\mathbf{c}}$ as functions of $\hat{\mathbf{m}}_{\mathbf{a}}$ and $\hat{\mathbf{m}}_{\mathbf{c}}$ to explicitly indicate that their magnitudes do not vary. The field lines generated by the point-dipole model are shown in Fig.2(a). Equation (1) exactly predicts the field produced by a spherical magnet and is an approximation for every other geometry that becomes more accurate with increasing distance [9]. The geometry of a nonspherical magnet can be adjusted to make (1) a more accurate approximation in the near-field [1]. In this section, we assume that the dipole field accurately models the field of the actuator magnet. (a) Dipole field lines and normalized magnitude $\|\mathbf{h}\| /\left\|\mathbf{m}_{\mathbf{a}}\right\|(\mathrm{mA} / \mathrm{m})$

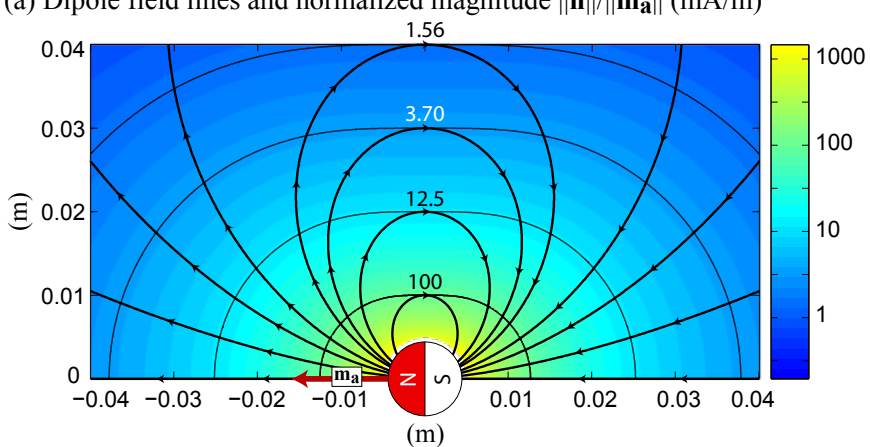

(b) Dipole force lines and normalized magnitude $\left\|\mathbf{f}_{\mathbf{m}}\right\| /\left(\left\|\mathbf{m}_{\mathbf{a}}\right\|\left\|\mathbf{m}_{\mathbf{c}}\right\|\right)(\mathrm{mN})$

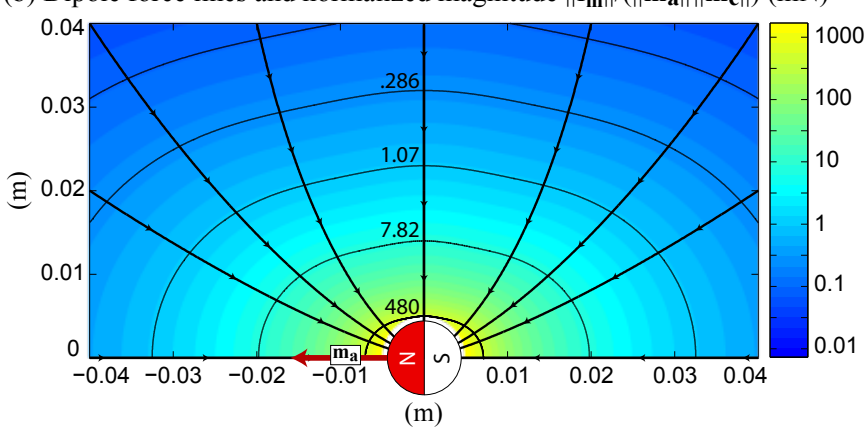

Fig. 2. Dipole field lines and the normalized actuator field magnitude $\|\mathbf{h}\| /\left\|\mathbf{m}_{\mathbf{a}}\right\|$ are shown in (a). Magnetic force lines, generated with (6), assuming the capsule's moment $\mathbf{m}_{\mathbf{c}}$ is aligned with the applied dipole field $\mathbf{h}$, and the resulting normalized magnetic force magnitude $\left\|\mathbf{f}_{\mathbf{m}}\right\| /\left(\left\|\mathbf{m}_{\mathbf{a}}\right\|\left\|\mathbf{m}_{\mathbf{c}}\right\|\right)$, are shown in (b). When the capsule's dipole moment is aligned with the applied magnetic field, the magnetic force tends to be attractive. The color scale is logarithmic in both plots.

The robot manipulator's $n$ revolute and prismatic joint velocities $\dot{\mathbf{q}} \in \mathbb{R}^{n}$ are mapped to the actuator magnet's spatial $\dot{\mathbf{p}}_{\mathbf{a}}$ and angular $\boldsymbol{\omega}_{\mathrm{a}}$ velocities by the robot manipulator Jacobian matrix $J_{\mathcal{R}}(\mathbf{q}) \in \mathbb{R}^{6 \times n}$ :

$$
\left[\begin{array}{c}
\dot{\mathbf{p}}_{\mathbf{a}} \\
\omega_{\mathrm{a}}
\end{array}\right]=J_{\mathcal{R}}(\mathbf{q}) \dot{\mathbf{q}}
$$

The point-dipole field (1) is radially symmetric about the actuator dipole moment and any component of $\boldsymbol{\omega}_{\mathrm{a}}$ in the direction of $\hat{\mathbf{m}}_{\mathbf{a}}$ produces no change in the magnetic field applied to the capsule. As a result, the robot manipulator Jacobian $J_{\mathcal{R}}(\mathbf{q})$ can be converted into an actuator-magnet Jacobian matrix $J_{\mathcal{A}}(\mathbf{q})$ that maps manipulator joint velocity $\dot{\mathbf{q}}$ to the actuator magnet's spatial velocity $\dot{\mathbf{p}}_{\mathbf{a}}$ and the actuator dipole moment's directional velocity $\dot{\hat{\mathbf{m}}}_{\mathrm{a}}=\boldsymbol{\omega}_{\mathrm{a}} \times \hat{\mathbf{m}}_{\mathrm{a}}$, with no contribution from the component of $\boldsymbol{\omega}_{\mathrm{a}}$ parallel to $\hat{\mathbf{m}}_{\mathbf{a}}$, by

$$
\left[\begin{array}{c}
\dot{\mathbf{p}}_{\mathbf{a}} \\
\dot{\hat{\mathbf{m}}}_{\mathbf{a}}
\end{array}\right]=\left[\begin{array}{cc}
I & 0 \\
0 & S\left(\hat{\mathbf{m}}_{\mathbf{a}}\right)^{\top}
\end{array}\right] J_{\mathcal{R}}(\mathbf{q}) \dot{\mathbf{q}}=J_{\mathcal{A}}(\mathbf{q}) \dot{\mathbf{q}}
$$

where $S\left(\hat{\mathbf{m}}_{\mathbf{a}}\right) \in s o(3)$ is the skew-symmetric form of the cross-product operation. The matrix $J_{\mathcal{A}}(\mathbf{q})$ can be used to approximately map small changes in the manipulator's joints to small changes in actuator-magnet position and small changes in the heading of the actuator magnet's dipole moment:

$$
\left[\begin{array}{c}
\delta \mathbf{p}_{\mathbf{a}} \\
\delta \hat{\mathbf{m}}_{\mathrm{a}}
\end{array}\right] \approx J_{\mathcal{A}}(\mathbf{q}) \delta \mathbf{q}
$$


Note that $J_{\mathcal{A}}(\mathbf{q})$ is not invertible and is at most rank five.

When the capsule is placed in the magnetic dipole field (1) generated by the actuator magnet, a magnetic torque $\tau_{\mathbf{m}}=$ $\mu_{0} \mathbf{m}_{\mathbf{c}} \times \mathbf{h}\left(\mathbf{p}, \hat{\mathbf{m}}_{\mathbf{a}}\right)$ and force $\mathbf{f}_{\mathbf{m}}=\mu_{0}\left(\mathbf{m}_{\mathbf{c}} \cdot \nabla\right) \mathbf{h}\left(\mathbf{p}, \hat{\mathbf{m}}_{\mathbf{a}}\right)$ are applied to the capsule's magnet, which are given by

$$
\begin{aligned}
\boldsymbol{\tau}_{\mathbf{m}}\left(\mathbf{p}, \hat{\mathbf{m}}_{\mathbf{a}}, \hat{\mathbf{m}}_{\mathbf{c}}\right)= & \frac{\mu_{0}\left\|\mathbf{m}_{\mathbf{a}}\right\|\left\|\mathbf{m}_{\mathbf{c}}\right\|}{4 \pi\|\mathbf{p}\|^{3}} \hat{\mathbf{m}}_{\mathbf{c}} \times D(\hat{\mathbf{p}}) \hat{\mathbf{m}}_{\mathbf{a}} \\
\mathbf{f}_{\mathbf{m}}\left(\mathbf{p}, \hat{\mathbf{m}}_{\mathbf{a}}, \hat{\mathbf{m}}_{\mathbf{c}}\right)= & \frac{3 \mu_{0}\left\|\mathbf{m}_{\mathbf{a}}\right\|\left\|\mathbf{m}_{\mathbf{c}}\right\|}{4 \pi\|\mathbf{p}\|^{4}}\left(\hat{\mathbf{m}}_{\mathbf{a}} \hat{\mathbf{m}}_{\mathbf{c}}^{\top}\right. \\
& \left.+\hat{\mathbf{m}}_{\mathbf{c}} \hat{\mathbf{m}}_{\mathbf{a}}^{\top}+\left(\hat{\mathbf{m}}_{\mathbf{c}}^{\top} Z \hat{\mathbf{m}}_{\mathbf{a}}\right) I\right) \hat{\mathbf{p}},
\end{aligned}
$$

where $Z=I-5 \hat{\mathbf{p}} \hat{\mathbf{p}}^{\top}$ and $\mu_{0}=4 \pi \times 10^{-7} \mathrm{~N} \cdot \mathrm{A}^{-2}$ is the permeability of free-space. The magnetic torque aligns the capsule's dipole moment with the applied field, while the magnetic force pulls the capsule in a direction determined by the field's spatial derivatives and the capsule's dipole moment.

When the magnetic capsule is actuated in fluid at low speeds, small accelerations, and without contact with other objects, there is little resistance to change in the capsule's heading, which enables the magnetic torque to quickly align the capsule's dipole moment with the applied field. In these conditions, we can assume that the capsule's dipole moment is approximately aligned with the applied field for all time:

$$
\hat{\mathbf{m}}_{\mathbf{c}}\left(\mathbf{p}, \hat{\mathbf{m}}_{\mathbf{a}}\right) \approx \hat{\mathbf{h}}\left(\mathbf{p}, \hat{\mathbf{m}}_{\mathbf{a}}\right)=\widehat{D(\hat{\mathbf{p}}) \hat{\mathbf{m}}_{\mathbf{a}}},
$$

and the capsule's heading can be controlled by adjusting the direction of the magnetic field without controlling the magnetic torque directly using (5), which would require measurement of the direction of $\hat{\mathbf{m}}_{\mathbf{c}}$ (i.e., the capsule's heading). It also implies that $\hat{\mathbf{m}}_{\mathbf{c}}$ can be predicted by (7) using only a measurement of the position $\mathbf{p}$ obtained by a localization system, and that the magnetic force applied to the magnetic capsule can be predicted by substituting (7) into (6):

$$
\begin{aligned}
\mathbf{f}_{\mathbf{m}}\left(\mathbf{p}, \hat{\mathbf{m}}_{\mathbf{a}}\right) \approx & \frac{3 \mu_{0}\left\|\mathbf{m}_{\mathbf{a}}\right\|\left\|\mathbf{m}_{\mathbf{c}}\right\|}{4 \pi\|\mathbf{p}\|^{4}\left\|D(\hat{\mathbf{p}}) \hat{\mathbf{m}}_{\mathbf{a}}\right\|}\left(\hat{\mathbf{m}}_{\mathbf{a}} \hat{\mathbf{m}}_{\mathbf{a}}^{\top}\right. \\
& \left.-\left(1+4\left(\hat{\mathbf{m}}_{\mathbf{a}}^{\top} \hat{\mathbf{p}}\right)^{2}\right) I\right) \hat{\mathbf{p}} .
\end{aligned}
$$

Note that 5-DOF magnetic control can be performed without making the low-speed, small-acceleration, and non-contact assumptions if a 5-DOF measurement of the capsule's pose (i.e., 3-DOF position and 2-DOF orientation of the capsule's dipole moment) is available. In this case, the applied magnetic force $f_{m}$ and the direction of the applied magnetic torque $\hat{\tau}_{m}$ can be controlled independently (the torque magnitude cannot be controlled independently of the force magnitude). As we will demonstrate, 5-DOF control can still be achieved using only 3-DOF measurement of the capsule's position.

The total force $\mathbf{f}$ applied to the capsule consists of the apparent weight $\mathbf{f}_{\mathrm{w}}$ (sum of the capsule's weight and buoyant force), which is constant, and the magnetic force $f_{m}$. We assume that the capsule is heavier than its buoyant force, making $\mathbf{f}_{\mathbf{w}}$ point in the direction of gravity. In this case, the capsule can be made to levitate by positioning the actuator magnet above the capsule, where the attractive magnetic force perfectly balances the capsule's apparent weight and the magnitude of the total applied force $\mathbf{f}$ is zero. If the capsule is desired to ascend, then the actuator magnet is moved closer so that the magnetic force is larger than the capsule's apparent weight and the total applied force is directed upward. If the capsule is desired to descend, then the actuator magnet is positioned farther away from the capsule's levitation position and the total applied force points down. The maximum downward force that can be applied is the capsule's apparent weight $\mathbf{f}_{\mathrm{w}}$.

Using (7) and (8), a nonlinear magnetic actuation equation can be formed that relates the relative position $\mathbf{p}$ and the direction of the actuator magnet $\hat{\mathbf{m}}_{\mathbf{a}}$ to the total applied force $\mathbf{f}$ and the direction of the applied magnetic field $\hat{\mathbf{h}}$ :

$$
\left.\left[\begin{array}{l}
\mathbf{f} \\
\hat{\mathbf{h}}
\end{array}\right]=\left[\begin{array}{c}
\mathbf{f}_{\mathbf{m}}\left(\mathbf{p}, \hat{\mathbf{m}}_{\mathbf{a}}\right) \\
\overline{D(\hat{\mathbf{p}}) \hat{\mathbf{m}}_{\mathbf{a}}}
\end{array}\right]=\mathcal{\mathbf { f } _ { \mathbf { w } }}\right]=\mathcal{F}\left(\mathbf{p}, \hat{\mathbf{m}}_{\mathbf{a}}\right)
$$

which is purely a function of the actuator magnet's pose, that is, the relative position $\mathbf{p}$ and the actuator magnet's dipole moment direction $\hat{\mathbf{m}}_{\mathbf{a}}$, which in turn, are purely specified by the capsule's position $\mathbf{p}_{\mathbf{c}}$ and the robot manipulator's pose $\mathbf{q}$.

In order to solve the "inverse" problem (i.e., computing the necessary manipulator pose that will apply a desired total applied force and an applied magnetic field heading, given the capsule's position), the nonlinear actuation equation (9) is first linearized with the Jacobian matrix $J_{\mathcal{F}} \in \mathbb{R}^{6 \times 6}$, computed by differentiating (9) with respect to the relative position $\mathbf{p}$ and the actuator dipole moment $\hat{\mathbf{m}}_{\mathbf{a}}$. Linearization produces the approximate mapping between small changes in relative position and actuator moment direction to small changes in the applied force and field heading:

$$
\begin{aligned}
{\left[\begin{array}{c}
\delta \mathbf{f} \\
\delta \hat{\mathbf{h}}
\end{array}\right] } & \approx J_{\mathcal{F}}\left(\mathbf{p}, \hat{\mathbf{m}}_{\mathbf{a}}\right)\left[\begin{array}{c}
\delta \mathbf{p} \\
\delta \hat{\mathbf{m}}_{\mathbf{a}}
\end{array}\right] \\
& =J_{\mathcal{F}}\left(\mathbf{p}, \hat{\mathbf{m}}_{\mathbf{a}}\right)\left(\left[\begin{array}{c}
\delta \mathbf{p}_{\mathbf{c}} \\
\mathbf{0}
\end{array}\right]+\left[\begin{array}{cc}
-I & 0 \\
0 & I
\end{array}\right]\left[\begin{array}{c}
\delta \mathbf{p}_{\mathbf{a}} \\
\delta \hat{\mathbf{m}}_{\mathbf{a}}
\end{array}\right]\right),
\end{aligned}
$$

where (11) results from substituting $\delta \mathbf{p}=\delta \mathbf{p}_{\mathbf{c}}-\delta \mathbf{p}_{\mathbf{a}}$ into (10). The relation (11) divides a small change in applied total force and applied field heading into the result of a small change in capsule position $\delta \mathbf{p}_{\mathbf{c}}$ and a small change in the actuator magnet's pose (i.e., the actuator-magnet position $\delta \mathbf{p}_{\mathbf{a}}$ and dipole heading $\delta \hat{\mathbf{m}}_{\mathbf{a}}$ ), which is related to small changes in the manipulator's joints by the Jacobian $J_{\mathcal{A}}$ (4).

Substituting (4) into (11) produces the relationship between small changes in the manipulator's joints and capsule position to small changes in applied total force and field heading:

$$
\begin{aligned}
& {\left[\begin{array}{l}
\delta \mathbf{f} \\
\delta \hat{\mathbf{h}}
\end{array}\right] \approx J_{\mathcal{F} \mathcal{A}}(\mathbf{p}, \mathbf{q}) \delta \mathbf{q}+J_{\mathcal{F}}\left(\mathbf{p}, \hat{\mathbf{m}}_{\mathbf{a}}\right)\left[\begin{array}{c}
\delta \mathbf{p}_{\mathbf{c}} \\
\mathbf{0}
\end{array}\right]} \\
& J_{\mathcal{F} \mathcal{A}}(\mathbf{p}, \mathbf{q})=J_{\mathcal{F}}\left(\mathbf{p}, \hat{\mathbf{m}}_{\mathbf{a}}\right)\left[\begin{array}{cc}
-I & 0 \\
0 & I
\end{array}\right] J_{\mathcal{A}}(\mathbf{q}) .
\end{aligned}
$$

The actuator magnet's dipole moment $\hat{\mathbf{m}}_{\mathbf{a}}$ does not appear in the arguments of $J_{\mathcal{F A}}$ since $\hat{\mathbf{m}}_{\mathbf{a}}$ is set by the robot manipulator's joints q using the manipulator's forward kinematics.

Equation (12) can be used inside a control loop where small changes in capsule position $\delta \mathbf{p}_{\mathbf{c}}$ are obtained by a capsule 
localization system, and $\delta \mathbf{f}$ and $\delta \hat{\mathbf{h}}$ are small desired changes produced by a controller governing the magnetic capsule's pose. In this context, 12 can be rearranged to produce

$$
\delta \mathbf{d}=\left[\begin{array}{l}
\delta \mathbf{f} \\
\delta \hat{\mathbf{h}}
\end{array}\right]-J_{\mathcal{F}}\left(\mathbf{p}, \hat{\mathbf{m}}_{\mathbf{a}}\right)\left[\begin{array}{c}
\delta \mathbf{p}_{\mathbf{c}} \\
\mathbf{0}
\end{array}\right] \approx J_{\mathcal{F A}}(\mathbf{p}, \mathbf{q}) \delta \mathbf{q},
$$

where $\delta \mathbf{d}$ is a desired change in applied force and field heading resulting only from a change in the manipulator's joints. Equation (14) can be inverted to produce the inverse mapping of desired change in applied force and a change in field heading to a necessary change in the manipulator's joints using the Moore-Penrose pseudoinverse:

$$
\delta \mathbf{q} \approx J_{\mathcal{F A}}(\mathbf{p}, \mathbf{q})^{\dagger} \delta \mathbf{d} .
$$

If multiple solutions of (15) are possible (i.e., the manipulator has more than 5-DOF), then the pseudoinverse solves (15) and minimizes $\|\delta \mathbf{q}\|$. (A generalized pseudoinverse can be applied for a manipulator where the units of $\delta \mathbf{q}$ are inconsistent [18].) Given an initial joint configuration $\mathbf{q}_{0}$, (15) can be integrated in time to produce $\mathbf{q}_{t}$ without explicitly solving the inverse kinematics of the complete manipulator-magnet system [20]. This approach breaks down when the manipulator is near a kinematic singularity, which we will address later in this paper.

\section{Analyzing the Jacobian $J_{\mathcal{F A}}(\mathbf{p}, \mathbf{q})$}

For 5-DOF holonomic control, $J_{\mathcal{F} \mathcal{A}}(\mathbf{p}, \mathbf{q})$ must be rank five. Since $J_{\mathcal{F A}}(\mathbf{p}, \mathbf{q})$ is the product of $J_{\mathcal{F}}\left(\mathbf{p}, \hat{\mathbf{m}}_{\mathbf{a}}\right)$ and $J_{\mathcal{A}}(\mathbf{q})$, we will analyze the rank of the Jacobian $J_{\mathcal{F}}\left(\mathbf{p}, \hat{\mathbf{m}}_{\mathbf{a}}\right)$ and the Jacobian $J_{\mathcal{A}}(\mathbf{q})$ separately. For readability, we will refer to the Jacobians $J_{\mathcal{F} \mathcal{A}}(\mathbf{p}, \mathbf{q}), J_{\mathcal{F}}\left(\mathbf{p}, \hat{\mathbf{m}}_{\mathbf{a}}\right)$ and $J_{\mathcal{A}}(\mathbf{q})$ without their arguments in the text (i.e., as $J_{\mathcal{F} \mathcal{A}}, J_{\mathcal{F}}$, and $J_{\mathcal{A}}$ ).

Prior to analyzing the rank of $J_{\mathcal{F}}$, we first scale the columns and rows of $J_{\mathcal{F}}$ to produce a nondimensional Jacobian $\widetilde{J}_{\mathcal{F}}$ that approximately maps its preimage, consisting of nondimensional changes in position $\delta \mathbf{p} /\|\mathbf{p}\|$ and changes in actuator magnet heading $\delta \hat{\mathbf{m}}_{\mathbf{a}}$ (already nondimensional), to its image, consisting of nondimensional changes in force $\delta \mathbf{f} /\left\|\mathbf{f}_{\mathbf{m}}\right\|$ and applied field heading $\delta \hat{\mathbf{h}}$ (already nondimensional):

$$
\widetilde{J}_{\mathcal{F}}\left(\mathbf{p}, \hat{\mathbf{m}}_{\mathbf{a}}\right)=\left[\begin{array}{cc}
\frac{1}{\left\|\mathbf{f}_{\mathbf{m}}\right\|} & 0 \\
0 & I
\end{array}\right] J_{\mathcal{F}}\left[\begin{array}{cc}
\|\mathbf{p}\| I & 0 \\
0 & I
\end{array}\right]
$$

where $I \in \mathbb{R}^{3 \times 3}$ is the identity matrix.

The nondimensional Jacobian $\widetilde{J}_{\mathcal{F}}$ is produced by post- and premultiplying $J_{\mathcal{F}}$ with a series of elementary matrices, which guarantees that $\operatorname{rank} \widetilde{J}_{\mathcal{F}}=\operatorname{rank} J_{\mathcal{F}}$ and enables the rank of $J_{\mathcal{F}}$ to be found using the singular value decomposition of $\widetilde{J}_{\mathcal{F}}$ with unit-consistent singular values, which reveal the rank of $J_{\mathcal{F}}$. Since the applied field direction $\hat{\mathbf{h}}$ cannot change in a direction parallel to itself, the smallest singular value $\sigma_{6}$ must be zero. The second smallest singular value $\sigma_{5}$ reveals whether the rank of rank $\widetilde{J}_{\mathcal{F}}=5$. Fig. 3 shows $\sigma_{5}$ plotted on a plane in which the actuator dipole moment $\mathbf{m}_{\mathbf{a}}$ lies. Because the magnetic field is radially symmetric about the dipole moment $\mathbf{m}_{\mathbf{a}}$, a plot of $\sigma_{5}$ on any plane in which $\mathbf{m}_{\mathbf{a}}$ lies will be equivalent to Fig. 3 The minimum value taken on by $\sigma_{5}$ is 0.123 , indicating that $\widetilde{J}_{\mathcal{F}}$ (and thus $J_{\mathcal{F}}$ ) is always rank five.

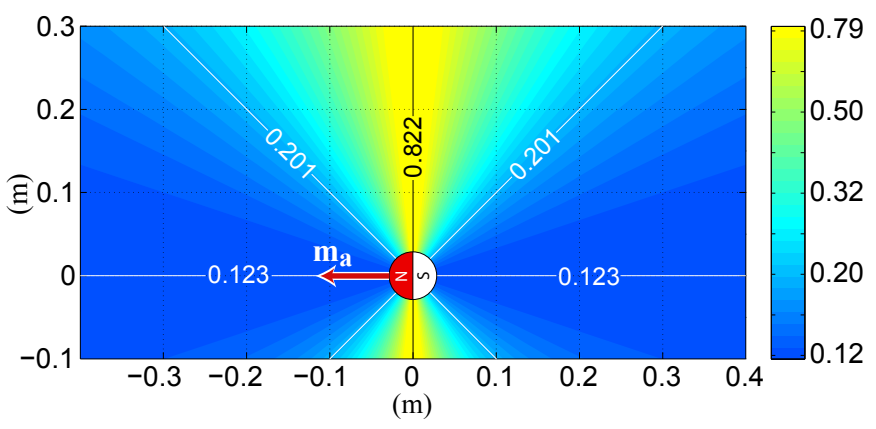

Fig. 3. The second smallest singular value, $\sigma_{5}$, of the normalized magnetic actuation Jacobian $\widetilde{J}_{\mathcal{F}}$ is plotted (with a logarithmic colorscale) on any plane in which the actuator dipole moment $\mathbf{m}_{\mathbf{a}}$ lies. The smallest value taken on by $\sigma_{5}$ is 0.123 and occurs on the line on which the dipole $\mathbf{m}_{\mathbf{a}}$ lies. The fact that $\sigma_{5}>0$ indicates that $\widetilde{J}_{\mathcal{F}}$ (and thus $J_{\mathcal{F}}$ ) is always rank five.

The fact that $J_{\mathcal{F}}$ is always rank five implies that a single permanent magnet in space, irrespective to the robot manipulator that maneuvers it, can exhibit 5-DOF control over a magnetic device. The ability of a complete robotic system, including magnet and manipulator, to exhibit 5-DOF magnetic control is precluded only by the ability of the robot manipulator to position the actuator magnet with 3-DOF and the actuator magnet's dipole moment with 2-DOF. If the rank of the Jacobian $J_{\mathcal{A}}$ is five, then the robotic system possesses 5-DOF control over the untethered capsule. If the actuator-magnet pose required to achieve a desired applied total force and magnetic field heading places the manipulator into a kinematic singularity, then 5-DOF magnetic control is lost.

We numerically analyze the configurations of total forces and field headings that make the manipulator enter a singularity by first nondimensionalizing the Jacobian $J_{\mathcal{A}}$ as

$$
\widetilde{J}_{\mathcal{A}}(\mathbf{q})=\left[\begin{array}{cc}
\frac{1}{\|\mathbf{p}\|} I & 0 \\
0 & I
\end{array}\right] J_{\mathcal{A}}(\mathbf{q})
$$

which can then be substituted, along with $\widetilde{J}_{\mathcal{F}}$, into 13) for $J_{\mathcal{F}}$ and $J_{\mathcal{A}}$ to produce the normalized Jacobian

$$
\widetilde{J}_{\mathcal{F A}}(\mathbf{p}, \mathbf{q})=\widetilde{J}_{\mathcal{F}}\left(\mathbf{p}, \hat{\mathbf{m}}_{\mathbf{a}}\right)\left[\begin{array}{cc}
-I & 0 \\
0 & I
\end{array}\right] \widetilde{J}_{\mathcal{A}},(\mathbf{q})
$$

which approximately maps change in manipulator joints $\delta \mathbf{q}$ (already nondimensional) to change in nondimensional applied force and change in field heading (already nondimensional).

The Moore-Penrose pseudoinverse $\widetilde{J}_{\mathcal{F} \mathcal{A}^{\dagger}}$ is the inverse mapping that minimizes $\|\delta \mathbf{q}\|$ if the robot manipulator is overactuated. The largest singular value of $\widetilde{J}_{\mathcal{F}} \mathcal{A}^{\dagger}$ (i.e., the reciprocal of the smallest nonzero singular value of $\widetilde{J}_{\mathcal{F A}}$ ) can be used to describe the worst case of how a unit-magnitude vector of nondimensional change in applied force and field heading are approximately mapped to a magnitude change in manipulator joints. If the largest singular value approaches infinity, then the robot manipulator is near a kinematic singularity.

As an example, consider the case where a capsule is desired to point downward (in the direction of gravity) as it is being repositioned by an applied magnetic force, and the manipulator 
(a) Largest singular value of $\widetilde{J}_{\mathcal{F A}}^{\dagger}$ in the $\hat{\mathbf{y}}, \hat{\mathbf{z}}$ plane

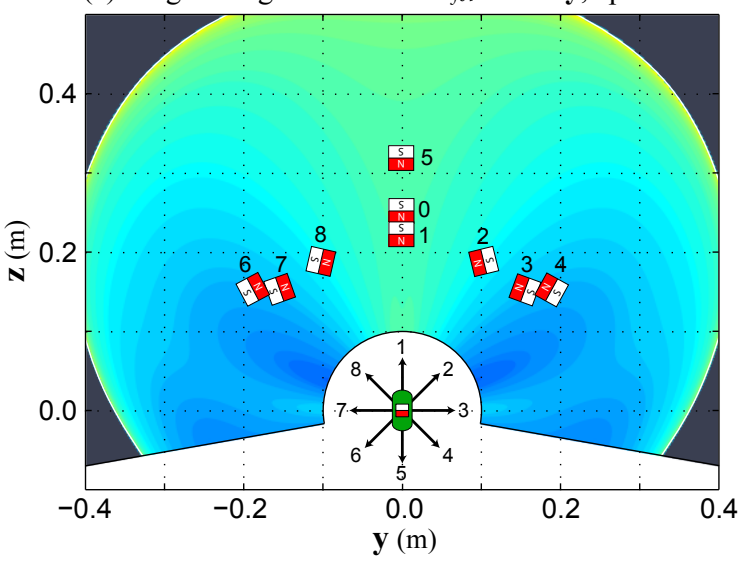

(b) Largest singular value of $\widetilde{J}_{\mathcal{F A}}^{\dagger}$ in the $\hat{\mathbf{x}}, \hat{\mathbf{z}}$ plane

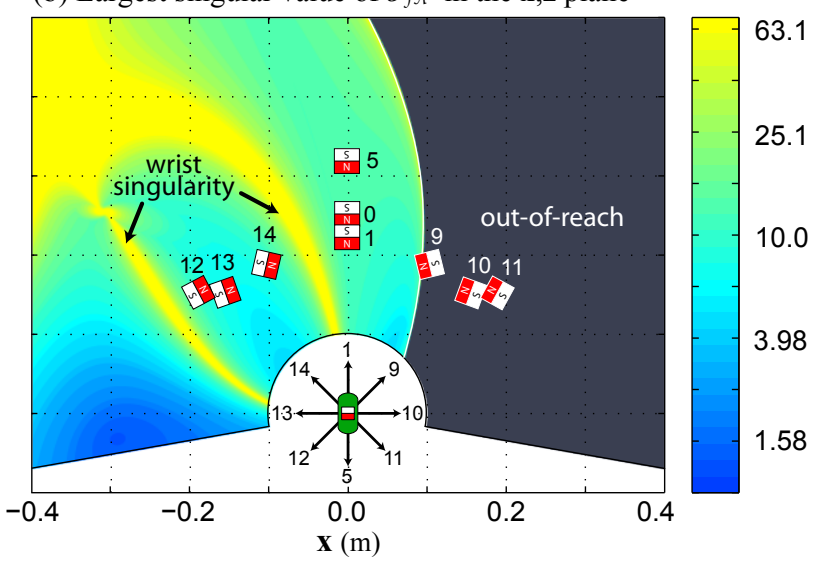

(c) Select robotic manipulator poses from (a) and (b)
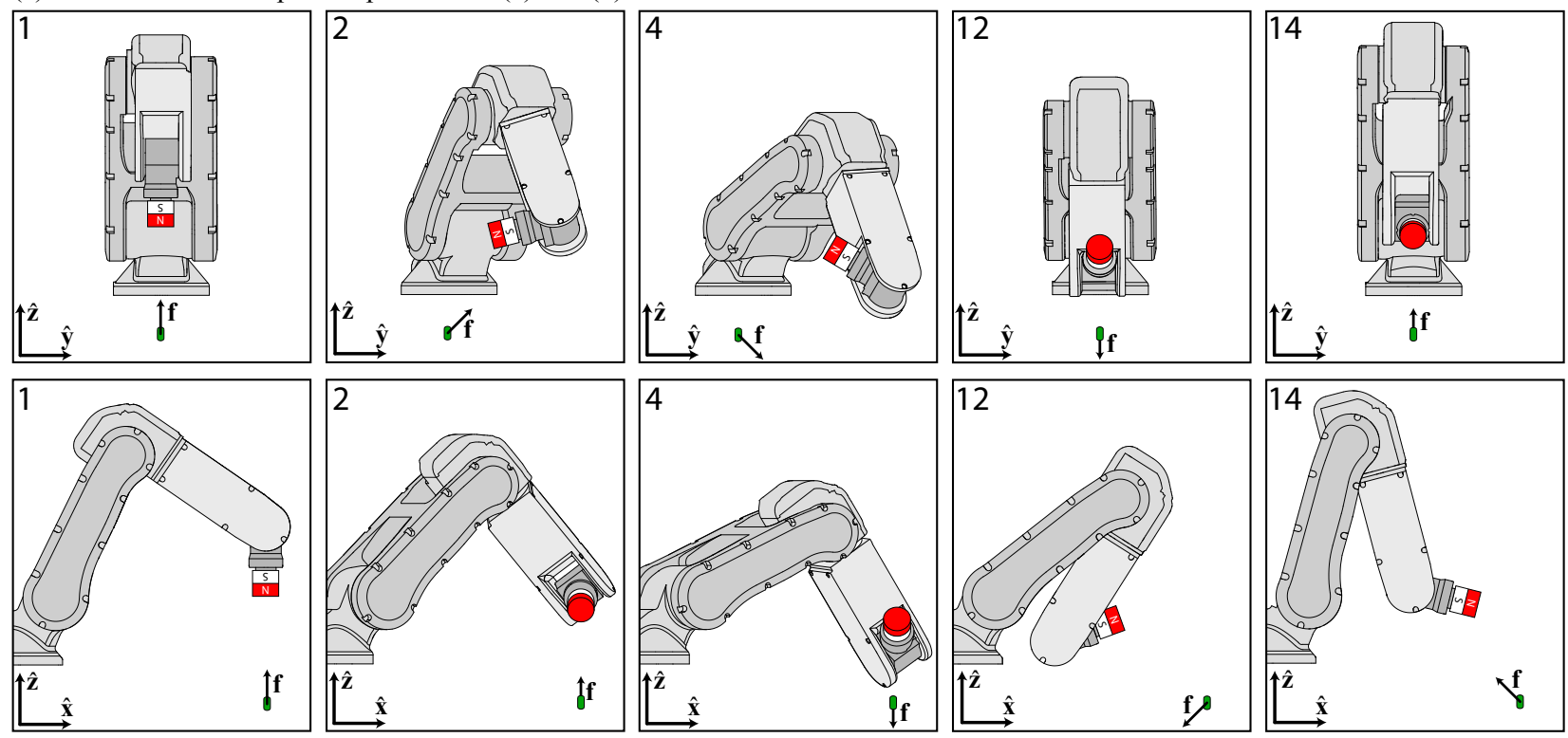

Fig. 4. The largest singular value of the nondimensional Jacobian $\widetilde{J}_{\mathcal{F A}}{ }^{\dagger}$ is shown in (a) the $\hat{\mathbf{y}}, \hat{\mathbf{z}}$ plane and in (b) the $\hat{\mathbf{x}}, \hat{\mathbf{z}}$ plane, plotted in a logarithmic colorscale, for a scenario where a capsule (positioned as shown) is desired to point downward $\left(\hat{\mathbf{m}}_{\mathbf{c}}=-\hat{\mathbf{z}}\right)$. The singular value plots are generated by placing the actuator magnet at every position in the $\hat{\mathbf{y}}, \hat{\mathbf{z}}$ and $\hat{\mathbf{x}}, \hat{\mathbf{z}}$ planes and directing the actuator magnet's dipole moment $\mathbf{m}_{\mathbf{a}}$ according to [19], so that the applied field at the capsule's position points the capsule down. The regions outside the reachable workspace of the manipulator are shown in gray. The regions where the largest singular value exceeds approximately 70 correspond to an actuator magnet pose that place the manipulator near a kinematic singularity. Fifteen numbered actuator poses are shown in (a) and (b) that correspond to the application of a correspondingly numbered $0.3 \mathrm{mN}$ total force acting on the capsule depicted in the figure. Pose 0 is the equilibrium position where the net applied force is zero. Front and left views of select manipulator configurations for five of the fifteen poses are shown in (c).

used to maneuver the actuator magnet is a 6-DOF serial link manipulator. For this example, we have assumed the capsule, actuator magnet, and manipulator are those used for the experiments herein and the capsule is placed in a typical position as illustrated in Fig.4 In Figs.4(a) and 4(b), the manipulator's workspace has been sliced into $\hat{\mathbf{x}}, \hat{\mathbf{z}}$ and $\hat{\mathbf{y}}, \hat{\mathbf{z}}$ planes. For every possible actuator-magnet position on both planes, the actuator magnet's direction $\hat{\mathbf{m}}_{\mathbf{a}}$ is set so that the field at the capsule position points in the $-\hat{\mathbf{z}}$ direction. The solution for $\hat{\mathbf{m}}_{\mathbf{a}}$ is given by

$$
\hat{\mathbf{m}}_{\mathbf{a}}=-\widehat{D^{-1}(\hat{\mathbf{p}})} \hat{\mathbf{z}}
$$

where $D^{-1}(\hat{\mathbf{p}})=(D(\hat{\mathbf{p}})-I) / 2$ admits a unique actuator magnet pose for every actuator magnet position [4].

The largest singular value of $\widetilde{J}_{\mathcal{F} \mathcal{A}^{\dagger}}$ resulting from the robot manipulator configuration that places the actuator magnet in every feasible position in the $\hat{\mathbf{x}}, \hat{\mathbf{z}}$ and $\hat{\mathbf{y}}, \hat{\mathbf{z}}$ planes and directs the actuator magnet's moment according to (19) are shown in Figs. 4(a) and 4(b). The regions where the manipulator nears its spherical-wrist kinematic singularity cause the singular value to become large. The regions outside the manipulator's reachable workspace are shown in gray.

Each actuator magnet pose causes a magnetic force to be applied to the capsule. Fifteen numbered actuator magnet poses are illustrated in Figs.4 a) and 4(b) along with the resulting correspondingly numbered total force vectors 
applied to the capsule. Each force vector denotes a total force magnitude of $\|\mathbf{f}\|=0.3 \mathrm{mN}$. The resulting manipulator poses for select numbered total force vectors are shown in Fig. 4(c). The pose that balances the applied magnetic force and the capsule's apparent weight is labeled as pose " 0 ". The manipulator's physical workspace limits and kinematic singularities complicate which forces the system can apply. For example, due to workspace limits of the manipulator, forces labeled "9," "10," and "11" are not achievable, and due to the manipulator's wrist singularity, transitions from the capsule levitation configuration (pose " 0 ") to a $0.3 \mathrm{mN}$ force in the $-\hat{\mathbf{x}}$ direction (pose "13") would require the manipulator to pass through its wrist singularity. Note that the direction of the actuator magnet's dipole moment satisfies (19) and applies a magnetic field in the $-\hat{\mathbf{z}}$ direction at the capsule's position.

\section{Managing Manipulator Singularities}

Managing a manipulator's motion near kinematic singularities, while applying differential kinematic inversion, is well studied [20]. We have implemented a strategy that sacrifices control over the capsule's heading in order to maintain control over the magnetic force applied to the capsule (thus its position) in the presence of a manipulator singularity. Sacrificing heading control transforms the complete magnetic manipulation system into one that is kinematically over-actuated.

Given a small desired change in applied field heading $\delta \hat{\mathbf{h}}_{\mathbf{d}}$ and a small desired change in applied magnetic force $\delta \mathbf{f}_{\mathbf{d}}$, the problem of sacrificing heading control, while maintaining control over the applied magnetic force, is posed as a constrained, quadratic least-squares problem, of the form

$$
\begin{array}{cl}
\underset{\delta \mathbf{q}}{\operatorname{minimize}} & \left\|\frac{\partial \hat{\mathbf{h}}}{\partial \mathbf{q}} \delta \mathbf{q}-\delta \hat{\mathbf{h}}_{\mathbf{d}}\right\| \\
\text { subject to } & \frac{\partial \mathbf{f}}{\partial \mathbf{q}} \delta \mathbf{q}=\delta \mathbf{f}_{\mathbf{d}} \\
& \|W \delta \mathbf{q}\| \leq r,
\end{array}
$$

which we solve numerically, where the matrices $\partial \mathbf{f} / \partial \mathbf{q} \in$ $\mathbb{R}^{3 \times n}$ and $\partial \hat{\mathbf{h}} / \partial \mathbf{q} \in \mathbb{R}^{3 \times n}$ are the top and bottom three rows of the Jacobian $J_{\mathcal{F A}}$, respectively. The constraint 21 guarantees the desired change in applied force $\delta \mathbf{f}_{\mathbf{d}}$ is met (provided $\partial \mathbf{f} / \partial \mathbf{q}$ has full row rank), and the constraint (22) enforces a maximum bound $r$ on the magnitude of joint motion, weighted by the invertible matrix $W$. The cost function 20 attempts to reduce the error between the desired and actual change in applied field heading. The weight matrix $W$ can be used to penalize select joint motions, to homogenize disparate units of $\delta \mathbf{q}$, or to keep the magnitude of $\delta \mathbf{q}$ within a "trust-region," where the Jacobian $J_{\mathcal{F A}}$ is accurate. Note that if the magnitude constraint (22) is inactive (e.g. if the robot manipulator is not near a kinematic singularity) and $J_{\mathcal{F} \mathcal{A}}$ is rank-five, then the solution to the formulation (20)-22) is equivalent to the solution obtained with the pseudoinverse (15).

There are two ways for the formulation 20 - 22 to break down. The first is if the matrix $\partial \mathbf{f} / \partial \mathbf{q}$ does not have full row rank and the constraint 21] is not satisfiable. The second is if the constraints (21) and (22) become mutually exclusive, which could occur if $\partial \mathbf{f} / \partial \mathbf{q}$ is ill-conditioned, $\left\|\delta \mathbf{f}_{\mathbf{d}}\right\|$ is too large, or $r$ is too small for the required joint motion $\delta \mathbf{q}$.

\section{EXPERIMENTAL RESUlts \& DisCUSSION}

The mockup capsule is actuated in a tank of water by an axially magnetized, grade $\mathrm{N} 42$, cylindrical $\mathrm{NdFeB}$ magnet with a height of $31.75 \mathrm{~mm}$, a diameter of $31.75 \mathrm{~mm}$, and with a dipole moment of $\left\|\mathbf{m}_{\mathbf{a}}\right\|=26.2 \mathrm{~A} \cdot \mathrm{m}^{2}$, positioned by a Yaskawa-Motoman MH5 6-DOF robotic manipulator. The capsule contains a cube $\mathrm{NdFeB}$ permanent magnet with its dipole moment $\left\|\mathbf{m}_{\mathbf{c}}\right\|=0.126 \mathrm{~A} \cdot \mathrm{m}^{2}$ arranged parallel to the capsule's principal axis; the remainder of the capsule's volume is filled with air. The capsule's weight is $15.3 \mathrm{mN}$ and the buoyancy force in water is $14.8 \mathrm{mN}$. The position of the capsule is triangulated by two orthogonal Basler A602FC cameras, which is used with an extended Kalman filter for capsuleposition feedback. Unless otherwise stated, the localization system's update frequency is $90 \mathrm{~Hz}$. The experimental setup, consisting of the robot manipulator, vision system, mockup capsule, and the actuator magnet is shown in Fig. 1.

A PID feedback controller (using the triangulated capsule position) with a gravity-compensating feedforward term, was implemented to servo the capsule to any desired position in the workspace. At every iteration, the PID controller takes as input a desired capsule position $\mathbf{p}_{\mathbf{c}, \mathbf{d}}$, an estimated capsule position $\overline{\mathbf{p}}_{\mathbf{c}}$, and an estimated capsule spatial velocity $\dot{\overline{\mathbf{p}}}_{\mathbf{c}}$, and produces a desired change in applied force $\delta \mathbf{f}$, which is then combined with a desired change in applied field heading $\delta \hat{\mathbf{h}}_{\mathbf{d}}$ and converted into robot manipulator motion by solving the constrained least-squares formulation $20-22$ with an identity weight matrix $W=I \in \mathbb{R}^{6 \times 6}$ unless otherwise noted. An estimate of the capsule's heading is obtained from the measured position using (1), and is controlled in an openloop fashion. In this paper, we compute a desired change in field heading (which is equivalent a desired change in capsule heading) as the difference between a desired capsule heading and an estimate of the current capsule heading, which we assume to be small at each controller iteration. Due to a limitation of the commercial manipulator control system, the robot manipulator's position is updated at $25 \mathrm{~Hz}$.

The theory presented herein, as well as our control system, is demonstrated by controlling the magnetic mockup capsule along multiple predefined trajectories. Fig.5.5) shows an image sequence of the capsule following a raster-scan trajectory where the capsule moves from right to left along a squarewave path with an amplitude of $25 \mathrm{~mm}$ and a period of $40 \mathrm{~mm}$. Such a trajectory could be used to perform automated visual coverage of a surface for inspection tasks. The desired squarewave trajectory and the actual paths traveled by the capsule while moving at desired spatial velocities of $2 \mathrm{~mm} / \mathrm{s}, 4 \mathrm{~mm} / \mathrm{s}$, $8 \mathrm{~mm} / \mathrm{s}$ and $16 \mathrm{~mm} / \mathrm{s}$ are shown in Fig.5.b). In general, the trajectory tracking performance is good at slow speeds and worsens with increasing desired spatial velocity. It is important to note that at high spatial velocities, a fluidic torque can be generated that may cause the capsule's dipole moment $\mathbf{m}_{\mathbf{c}}$ 
(a) Raster-scan trajectory with constant capsule heading
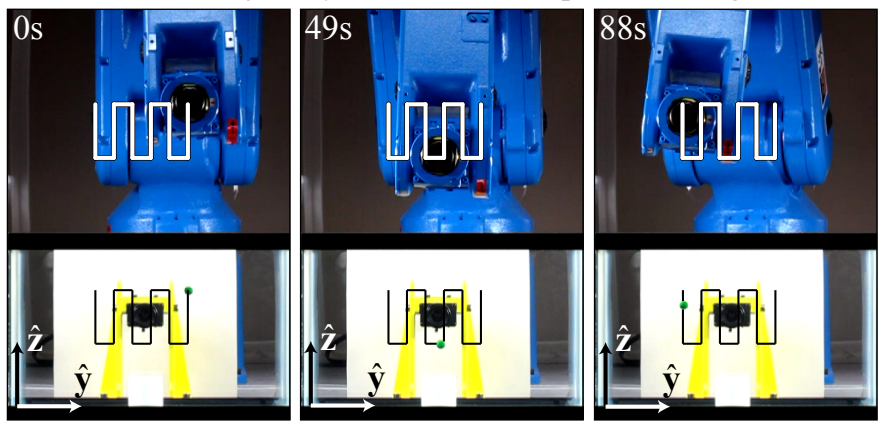

(b) Raster-scan trajectory tracking performance

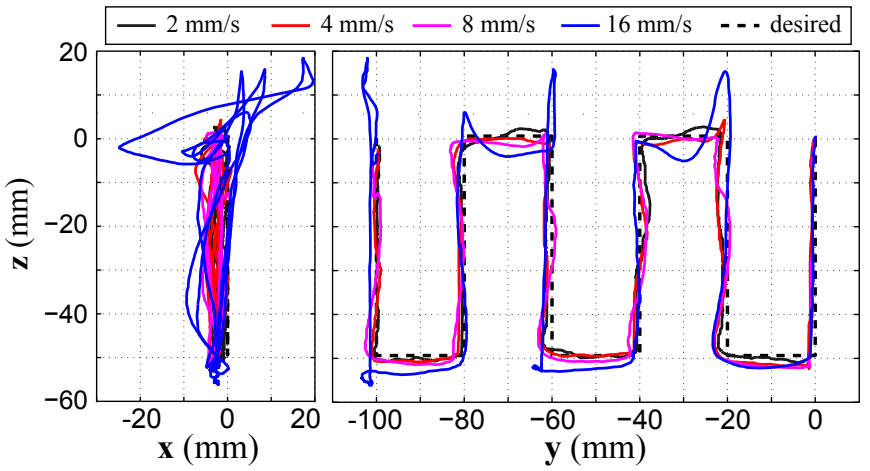

Fig. 5. An image sequence of the capsule completing a raster-scan trajectory (moving from right to left) with a desired capsule spatial velocity of $4 \mathrm{~mm} / \mathrm{s}$ is shown in (a), along with the approximate path taken by the actuator magnet. The capsule is oriented in the $\hat{\mathbf{x}}$ direction (toward the camera). The tracking performance of the control system for desired capsule spatial velocities ranging from $2 \mathrm{~mm} / \mathrm{s}$ to $16 \mathrm{~mm} / \mathrm{s}$ is shown in (b). With a desired spatial velocity of $16 \mathrm{~mm} / \mathrm{s}$, the raster-scan trajectory is completed in $35 \mathrm{~s}$.

to become misaligned with the applied magnetic field $\mathbf{h}$. If this occurs, then the assumptions of Sec. [I] are violated and the actual applied magnetic force may deviate from expected. This occurs to the capsule when following the square-wave trajectory with a desired spatial velocity of $16 \mathrm{~mm} / \mathrm{s}$, causing the capsule to deviate wildly from desired, which can be particularly observed in the $\hat{\mathbf{x}}, \hat{\mathbf{z}}$ plane.

The vision system used to track the 3-DOF capsule position is not feasible for clinical use. Existing clinically relevant localization strategies include RF triangulation [11], magnetic methods [7, 19, 21, 23], and CT scan or x-ray fluoroscopy [8]. In the previous experiments, the mockup capsule's position was localized at $90 \mathrm{~Hz}$ by the vision system. Clinically feasible localization methods may not provide the capsule's position at high rates (the method of [7] can perform 3D position-tracking at approximately $50 \mathrm{~Hz}$ ). The ability to actuate a mockup capsule with reduced 3D localization update frequencies is demonstrated in Fig.6, which shows the mockup capsule performing a remote-center-of-motion (RCM) maneuver, requiring the capsule's position to rotate around (while simultaneously pointing at) a fixed point in space. The update frequency of the tracking system (including the extended Kalman filter) was reduced to $60 \mathrm{~Hz}$ and $30 \mathrm{~Hz}$, in order to simulate the update rate of a more clinically relevant (a) Remote-center-of-motion (RCM) maneuver

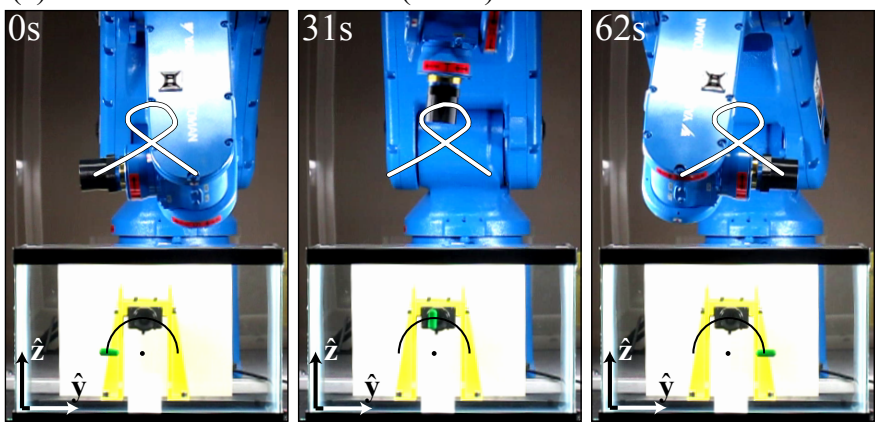

(b) RCM position tracking with varying localization rates

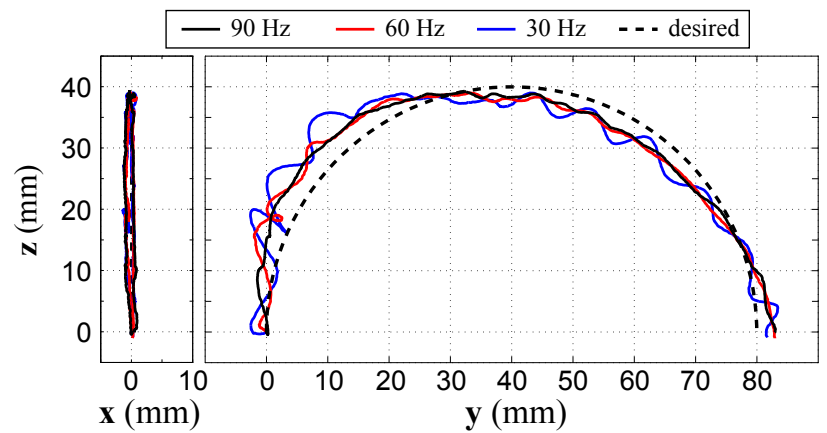

(c) RCM heading tracking with varying localization rates

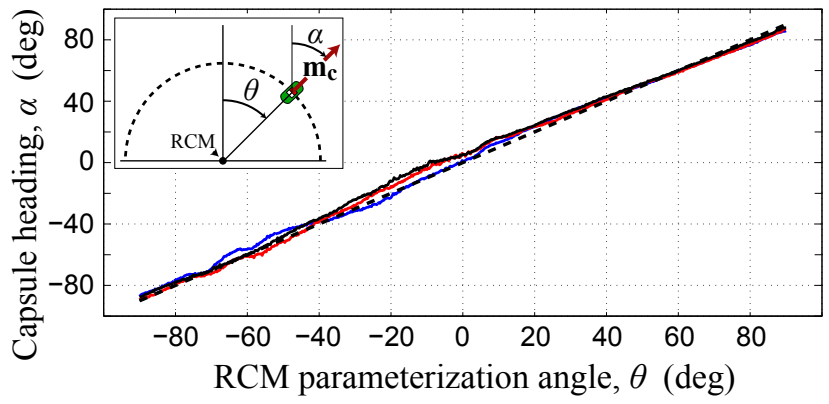

Fig. 6. The mockup capsule follows a desired remote-center-of-motion (RCM) maneuver in (a), with the approximate path taken by the actuator magnet shown. The position-following performance in the $\hat{\mathbf{x}}, \hat{\mathbf{z}}$ and $\hat{\mathbf{y}}, \hat{\mathbf{z}}$ planes, with the localization system tracking the capsule at 30,60 , and $90 \mathrm{~Hz}$, is shown in (b). The heading-following performance of the mockup capsule (measured in the $\hat{\mathbf{y}}, \hat{\mathbf{z}}$ plane) is shown in (c). The capsule heading is measured using the vision system but is controlled in an open-loop fashion.

localization method. Fig.6(a) shows images of the capsule performing the RCM maneuver with $90 \mathrm{~Hz}$ localization rate, while Fig.6(b) shows the 3D position-tracking performance of the capsule for localization rates of 30,60 , and $90 \mathrm{~Hz}$. The average position-tracking error for the localization rates of 30 , 60 , and $90 \mathrm{~Hz}$ is $2.8,2.2$, and $2.1 \mathrm{~mm}$, respectively, with a standard deviation of $1.8,1.2$, and $1.0 \mathrm{~mm}$, respectively. The capsule's heading-tracking performance is shown in Fig.6(c). The heading-tracking performance tends to vary less with the localization frequency. The capsule moves at approximately $2 \mathrm{~mm} / \mathrm{s}$ and completes the RCM maneuver in $62 \mathrm{~s}$.

Fig.7 (7) shows the capsule transitioning from the configuration that forces the robot manipulator to enter its wrist singularity, to a configuration where the capsule points in 
(a) Transitioning out of the manipulator wrist singularity
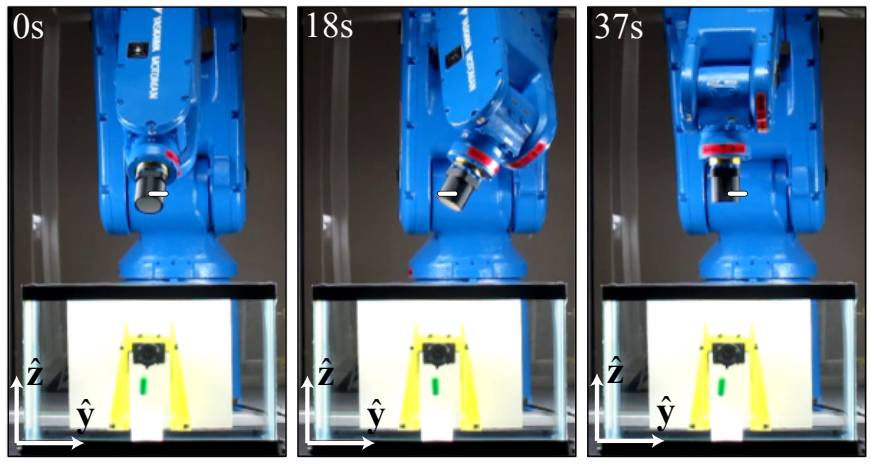

(b) U-shaped trajectory
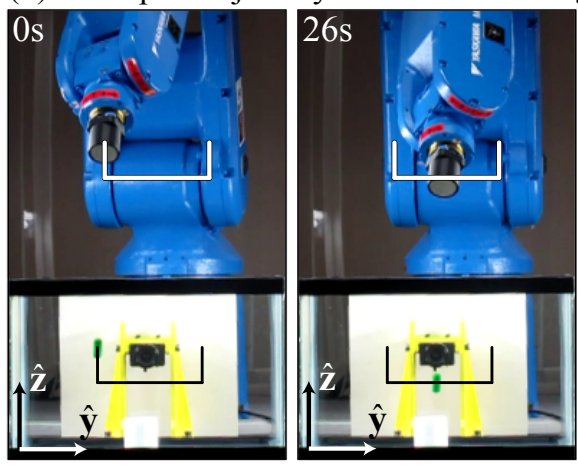

gularity

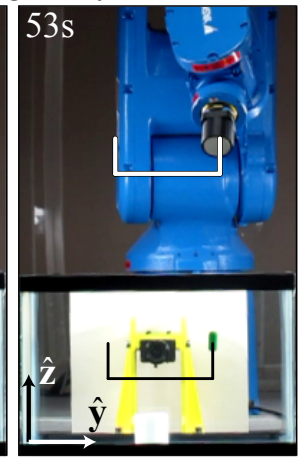

Fig. 7. In (a), the user commands the capsule to rotate from a configuration that forces the robot manipulator into its wrist singularity, to a configuration with the capsule pointed in the $-\hat{\mathbf{z}}$, while maintaining the capsule's position in space. Rather than immediately rotating the third joint to accommodate the desired capsule motion, the control method presented guides the manipulator to first rotate the capsule about the $\hat{\mathbf{z}}$ axis in order to reduce net manipulator motion, while maintaining control over the capsule's position. In (b), the capsule follows a U-shaped trajectory even with the manipulator near its wrist singularity, which demonstrates 3-DOF control over the capsule's position. The approximate path taken by the actuator magnet is shown in both examples.

the $-\hat{\mathbf{z}}$ direction by rotating the desired capsule direction $10^{\circ}$ around the $-\hat{\mathbf{y}}$ axis, while simultaneously keeping the capsule's position in space stationary. In this example, the weight matrix $W=\operatorname{diag}([1,1,1,20,1,1])$ and the bound $r=0.04$ radians, which penalizes motion in the manipulator's fourth joint more than the others. In the initial robot manipulator configuration at time $t=0 \mathrm{~s}$, the desired change in the capsule direction would require the fourth joint of the robot manipulator to rapidly rotate $\pi / 2$ radians. Rather than rapidly rotating the fourth joint, the constraint $\|W \delta \mathbf{q}\| \leq r$ penalizes the fourth joint's velocity and forces the manipulator to first rotate the capsule about the $\hat{\mathbf{z}}$ axis (which can be seen at $t=18 \mathrm{~s}$ ) before rotating about the $\hat{\mathbf{y}}$ axis as desired. This demonstrates the ability of the controller to balance desired changes in the capsule's configuration that may conflict with the robot manipulator's kinematics in a singularity.

Fig.7 b) shows the mockup capsule following a U-shaped trajectory, with the desired capsule dipole moment pointing in the direction that forces the robot manipulator into its wrist singularity. The robot manipulator's joint configuration is nearly in the wrist-singular configuration throughout the trajectory. In this demonstration, the Jacobian $J_{\mathcal{F} \mathcal{A}}$ is illconditioned and causes the inverse-kinematics approach using the psuedoinverse (15) to break down, which would result in incorrect magnetic forces being applied to the capsule. By solving the inverse kinematics using the formulation 20 (22), control over the applied magnetic force is maintained (as indicated by the successful completion of the desired trajectory) but at the sacrifice of the capsule's heading.

The stability of the mockup capsule under the control of the methods presented is difficult to study due to the nonlinearity of the applied magnetic field, nonlinearities resulting from the constraints of the formulation $20-22$, and in our case, velocity limitations of the manipulator. However, provided the magnetic torque dominates the mockup capsule's rotational dynamics and the actuator magnet is quasistatic, the applied magnetic torque will always stably align the capsule's dipole moment with the applied dipole field. In general, robotic manipulators tend to be bandwidth-limited. In the experiments, water provided damping that insures stability in the capsule's position. Actuating the capsule in air with little damping would be difficult using a commercial manipulator.

The volume of the actuator magnets plays a role in robustness. Since the applied magnetic force scales as $\|\mathbf{p}\|^{-4}$, small changes in the distance $\|\mathbf{p}\|$ cause a change in the magnetic force that scales as $\|\mathbf{p}\|^{-5}$, which becomes large when $\|\mathbf{p}\|$ is small. Increasing the size of the actuator and capsule magnets increases the magnitude of their dipole moments and allows the capsule to be actuated where $\|\mathbf{p}\|$ is greater, and the applied magnetic force is less sensitive to changes in $\|\mathbf{p}\|$. If the manipulator has velocity limits, then the actuator-magnet volume should be selected so that the manipulator's velocity is not exceeding when compensating for typical disturbances in the capsule's position. Future work should include further study of stability and robustness.

\section{CONCLUSION}

We have presented a method for 3-DOF position and 2-DOF orientation control of a mockup magnetic capsule endoscope using a single permanent magnet positioned by a 6-DOF seriallink manipulator, while only requiring the 3-DOF capsule position to be measured. We have studied the kinematics of manipulating an untethered magnetic device using a single permanent magnet as the end-effector of a robot manipulator, and introduced a control method that seamlessly sacrifices control over the capsule's heading in order to maintain control over the capsule's position when the robot manipulator enters a kinematic singularity. We have demonstrated our method's robustness to a control rate of $25 \mathrm{~Hz}$, a localization rate of $30 \mathrm{~Hz}$, deviation in the applied magnetic field from expected, and the presence of manipulator singularities. We find that the manipulator's kinematic singularities and workspace limitations are the most limiting factors to the methods presented herein. Although our method has application in magnetic capsule endoscopy of a fluid-distended stomach, the methods presented can be applied to magnetic manipulation in general. 


\section{REFERENCES}

[1] A. J. Petruska and J. J. Abbott. Permanent-magnet geometries for dipole field approximation. IEEE Trans. Magn., 49(2):811-819, 2013.

[2] A. W. Mahoney and J. J. Abbott. Managing magnetic force applied to a magnetic device by a rotating dipole field Appl. Phys. Lett., 99(134103):1-3, 2011.

[3] A. W. Mahoney and J. J. Abbott. 5-DOF manipulation of a magnetic capsule in fluid using a single permanent magnet: proof-of-concept for stomach endoscopy. In Proc. $6^{\text {th }}$ Hamlyn Symp. Medical Robotics, pages 114115, 2013.

[4] A. W. Mahoney and J. J. Abbott. Generating rotating magnetic fields with a single permanent magnet for propulsion of untethered magnetic devices in a lumen IEEE Trans. Robot., 30(2):411-420, 2014.

[5] B. J. Nelson, I. K. Kaliakatsos, and J. J. Abbott. Microrobots for minimally invasive medicine. Апnи. Rev. Biomed. Eng., 12:55-85, 2010.

[6] B. L. Nguyen,, J. L. Merino, and E. S. Gang. Remote navigation for ablation procedures - A new step forward in the treatment of cardiac arrthymias Eur. Cardiol., 6: 50-56, 2010.

[7] C. Di Natali, M. Beccani, and P. Valdastri. Real-time pose detection for magnetic medical devices. IEEE Trans. Magn., 49(7):3524-3527, 2013.

[8] F. Carpi and C. Pappone. Magnetic maneuvering of endoscopic capsules by means of a robotic navigation system. IEEE Trans. Biomed. Eng., 56(5):1482-1490, may 2009.

[9] E. P. Furlani. Permanent magnet and electromechanical devices: materials, analysis, and applications. Academic Press, San Diego, California, first edition, 2001.

[10] J. Keller et. al. Inspection of the human stomach using remote-controlled capsule endoscopy: a feasibility study in healthy volunteers (with videos) Gastrointest. Endosc., 73(1):22-28, 2011.

[11] D. Fischer, R. Schreiber, D. Levi, and R. Eliakim. Capsule Endoscopy: the localization system. Gastrointest. Endoscopy Clin. N. Am., 14(1):25-31, 2004.

[12] G. Ciuti, P. Valdastri, A. Menciassi, and P. Dario. Robotic magnetic steering and locomotion of capsule endoscope for diagnostic and surgical endoluminal procedures Robotica, 28(2):199-207, 2010.

[13] G. Ciuti, R. Donlin, P. Valdastri, A. Arezzo, A. Menciassi, M. Morino, and P. Dario. Robotic versus manual control in magnetic steering of an endoscopic capsule. Endoscopy, 42(2):148-152, 2010.

[14] G.-L. Jiang, Y.-H. Guu, C.-N. Lu, P.-K. Li, H.-M. Shen, L.-S. Lee, J. A. Yeh, and M. T. Hou. Development of rolling magnetic microrobots. J. Micromech. Microeng., 20:085042, 2010.

[15] G. Lien, C. Liu, J. Jiang, C. Chuang, and M. Teng. Magnetic control system targeted for capsule endoscopic operations in the stomach: design, fabrication, and in vitro and ex vivo evaluations IEEE Trans. Biomed. Eng., 59(7):2068-2079, 2012.

[16] H. Keller, A. Juloski, H. Kawano, M. Bechtold, A. Kimura, H. Takizawa, and R. Kuth. Method for navigation and control of a magnetically guided capsule endoscope in the human stomach. In Proc. IEEE Int. Conf. Biomedical Robotics and Biomechatronics, pages 859-865, 2012.

[17] J. Kim, Y. Kwon, and Y. Hong. Automated alignment of rotating magnetic field for inducing a continuous spiral motion on a capsule endoscope with a twistable thread mechanism Int. J. of Prec. Eng. Manu., 13(3):371-377, 2012.

[18] K. L. Doty, C. Melchiorri, and C. Bonivento. A theory of generalized inverses applied to robotics Int. J. Robot. Res., 12(1):1-19, 1993.

[19] K. M. Popek, A. W. Mahoney, and J. J. Abbott. Localization method for a magnetic capsule endoscope propelled by a rotating magnetic dipole field In IEEE Int. Conf. Robotics and Automation, pages 5328-5333, 2013.

[20] L. Sciavicco and B. Siciliano. Modeling and control of robot manipulators. Springer-Verlag, London, UK, second edition, 2000.

[21] M.-G. Kim, Y.-S. Hong, and E.-J. Lim. Position and orientation detection of capsule endoscopes in spiral motion. Int. J. of Prec. Eng. and Manuf., 11(1):31-37, 2010.

[22] M. P. Kummer, J. J. Abbott, B. E. Kratochvil, R. Borer, A. Sengul, and B. J. Nelson. OctoMag: An electromagnetic systems for 5-DOF wireless micromanipulation IEEE Trans. Robot., 26(6):1006-1017, 2010.

[23] M. Salerno, G. Ciuti, G. Lucarini, R. Rizzo, P. Valdastri, A. Menciassi, A. Landi, and P. Dario. A discrete-time localization method for capsule endoscopy based on onboard magnetic sensing Meas. Sci. Technol., 23(01570), 2012.

[24] M. T. Hou, H.-M. Shen, G.-L. Jiang, C.-N. Lu, I.-J. Hsu, and J. A. Yeh. A rolling locomotion method for untethered magnetic microrobots Appl. Phys. Lett., 96 (024102):1-3, 2010.

[25] P. Berkelman and M. Dzadovsky. Magnetic levitation over large translation and rotation ranges in all directions IEEE/ASME Trans. Mechatronics, 18(1):44-52, 2013.

[26] P. Valdastri, G. Ciuti, A. Verbeni, A. Menciassi, P. Dario, A. Arezzo, and M. Morino. Magnetic air capsule robotic system: proof of concept of a novel approach for painless colonoscopy Surg. Endosc., 26(5):1238-1246, 2012.

[27] P. Valdastri, M. Simi, and R. J. Webster III. Advanced Technologies for Gastrointestinal Endoscopy, Annu. Rev. Biomed. Eng., 14:397-429, 2012.

[28] S. Yim and M. Sitti. Design and rolling locomotion of a magnetically actuated soft capsule endoscope IEEE Trans. Robot., 28(1):183-194, 2012. 